\title{
VIDEO MAGNETIC FIEILD AND VELOCITY FIELDS OF \\ SOLAR FLARES AND RELATIVE ACTIVE REGION
}

\author{
Ai Guoxiang, Li Jing
}

Beijing Astronomical Obs. Chinese Academia of Sciences

By means of Solar Magnetic Field Telescope [.1], the fine structure of video magnetic fields (5324A) and sight-of-line velocity fields ( $4861 \mathrm{~A})$ of flare active region on July 23(E53, S19) and Aug. 8(W25, S28) in 1987 has been obtained. The main characters are following:

1. The flares occur in places where there are compressive and osmotic motion between opposite magnetic poles. In some causas, the osmotic opposite magnetic pole dissipates after the flares.

2. Sight-line velocity neutral lines are consistent with the magnetic neutral lines. But in the two ends of " $S$ " shape of magnetic neutral line, the directions of flow passing the magne:ic neutral line are opposite. It shows compressive and shearing motion in large scale of the active region. The flares occur near the flow neutral line where there are the most compressive and shearing motion ( See Fig.1. and Fig.2.).

3. Chromospheric flow show clearly fiber. The loop and ejective constructions are correspondence magnetic structures.

4. On the feet points of the flares there are down flow about 3--10 km/ Sec. (See Fig.3. and Fig.4.) 


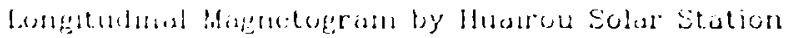

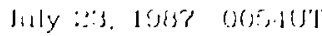

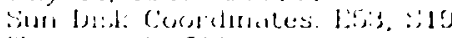

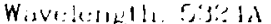

fromes.

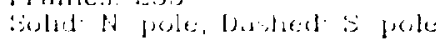

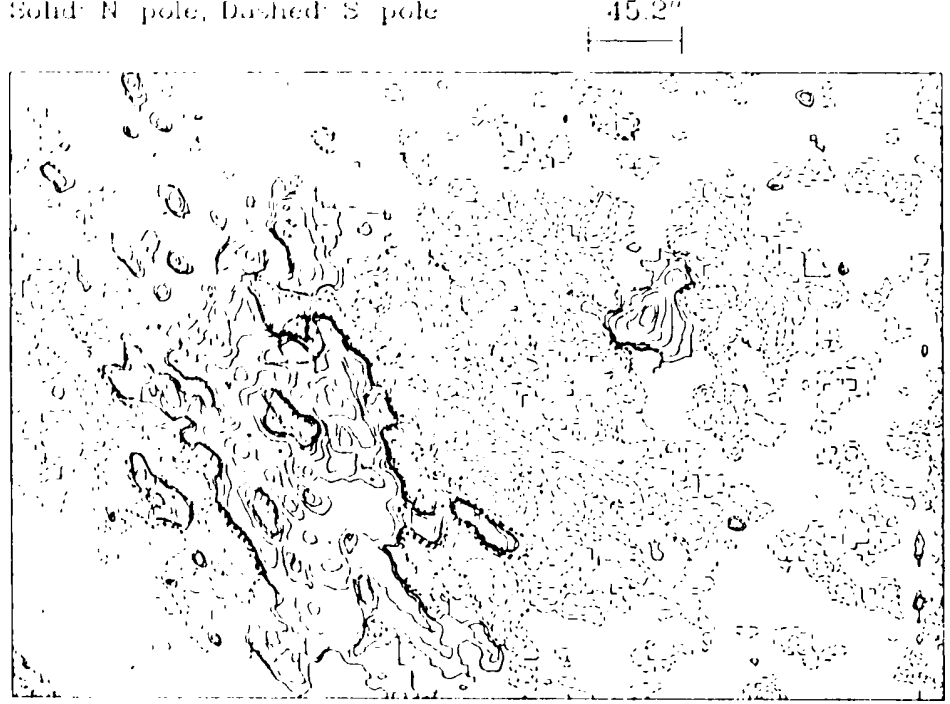

Fitel 'hle posilion of flare was traced by the circle

1..121s: $: 20,10,80,160,320,0 \cdot 10,960,1280,1600,1920,2210$

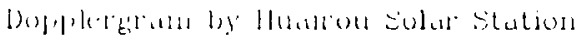

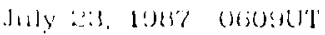

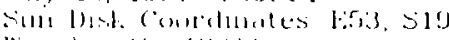

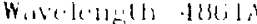

linatures

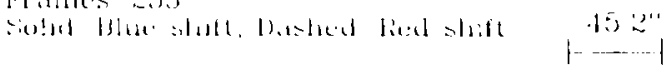

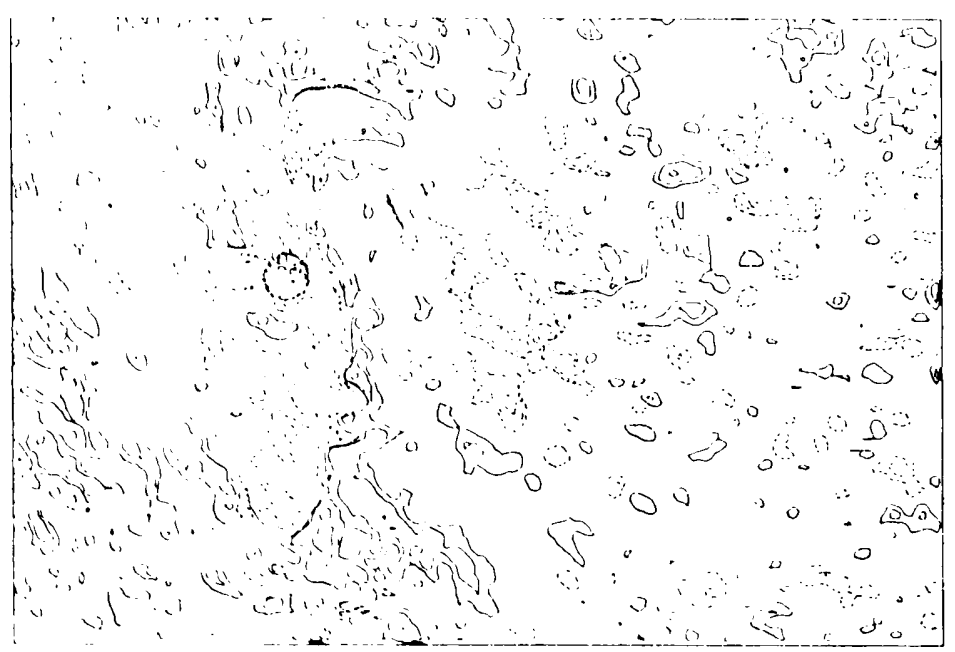

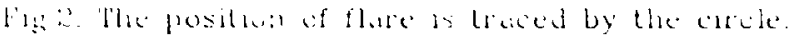

thie thestan of flow is down where flare vecured.

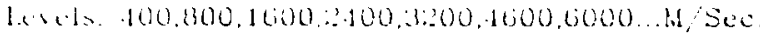


Longitudinal Magnetogram by lluairou Solar Station

August 06. 1367 003630?

Sun bisk Courdnates: Wids, size

Wivelength: S3r.at

Frames:

Solid: $N$-pole. Dastued: $s$-pule
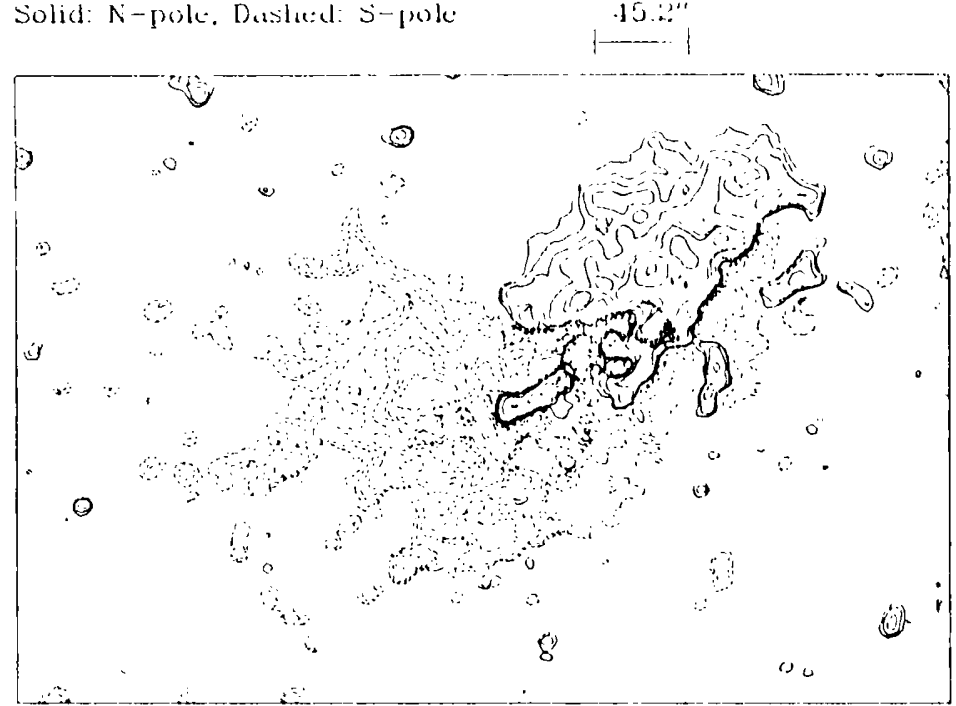

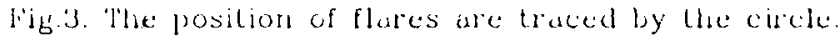

There: are lwo places cireled can be seen.

Levels: $20,40,80,160,320,6110,960,1230,1600,19,20,2210$

Dopslurgram by lluarrou solur Station

Alugust 00, 1937 O135U'

Sun Disk Cooldinutes: W25, S28

Wavelength: 1836$] \mathrm{A}$

Franus 255

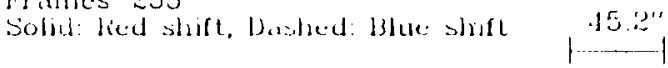

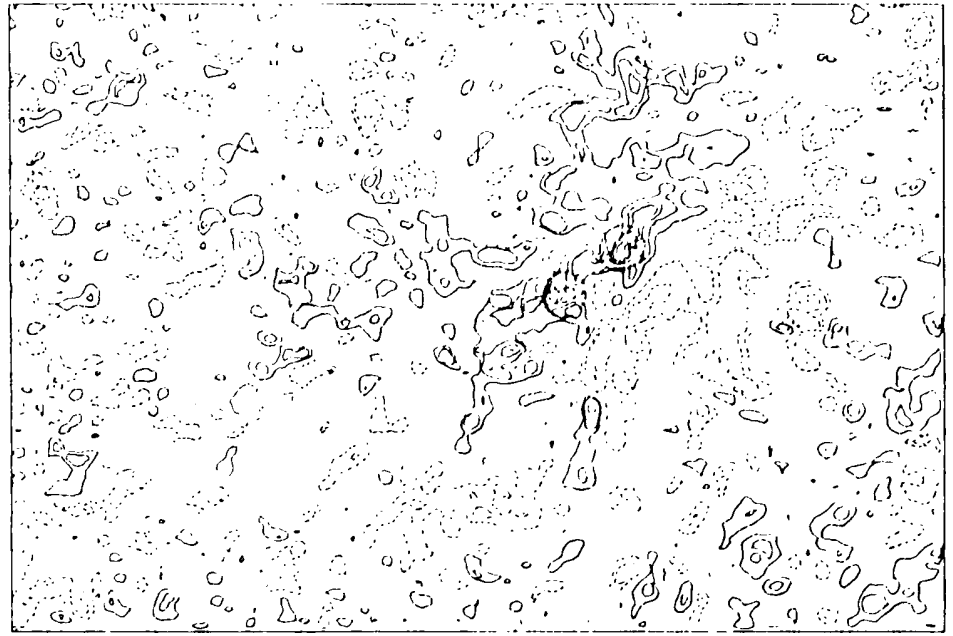

Pig.4. The position of flares are traced by the circle.

The flow have different dircetion where flares occured Levels: $100,800,1600,8100,3200,1600,6000 \ldots \mathrm{N} / \mathrm{Sec}$ 
Above characters show that the energy of the small flares come from txcessive magnetic energy of force-free magnetic field which are pruduced by the compression and osmotic motion of oppsity poles. There effects adjust potential analyses support the force-free field. These observations and analyses support the mode of flare by the compresive force-frue magnetic fields [2].

The constructions of magnetic and velocity fields of the small flares are consisted with that of the large flares, that may be inspiration to stellar flares with larger size and energy.

\section{References}

[1]. Ai Guoxiang and Hu Yuefeng, Acta Astronomica Sinica., Vol. 27, No.2, $(1986), 173$.

[2]. Ai Guoxiang and Kong Fanxi, Acta Astronomica Sinica., Vol.23. No.3, (1982), 211. 